\title{
Impact of climate-change scenarios on offshore wind turbine structural performance
}

\author{
Wilkie, D. ${ }^{1, *}$, Galasso C. ${ }^{1,2}$ \\ ${ }^{1}$ Department of Civil, Environmental \& Geomatic Engineering, University College London, London, UK \\ ${ }^{2}$ Scuola Universitaria Superiore (IUSS) Pavia, Pavia, Italy
}

\begin{abstract}
.
Offshore wind turbines (OWTs) must be sufficiently robust and resilient to withstand windstorms over an operational life of 20-25 years in an aggressive marine environment. Current performance-based assessment methods for OWTs often neglect structural failure and focus on equipment failure only, which can be assessed using existing empirical databases. This study uses a simulation-based approach to assess various performance metrics associated with offshore wind infrastructure exposed to operational wind and wave conditions. Surrogate modelling is used to predict structural failure due to fatigue in a computationally efficient manner. The proposed surrogate model is based on Gaussian process regression and allows one to run structural simulations at a small training sample of wind and wave conditions and emulates the response at combinations where the OWT was not explicitly assessed. This result in an integrated probabilistic performance-based assessment framework for OWTs that considers both structural and non-structural (equipment) components. In particular, the proposed framework is used to evaluate the potential impact of climate-change scenarios on various OWT performance metrics, namely, fatigue damage, fatigue reliability and, ultimately, financial losses (cost of direct damage) for a case study OWT. This is compared to the change in revenue resulting from power production to understand which is more sensitive to climate change. Both fatigue damage and structural safety are found to be sensitive to changes in the site environmental conditions. However, as financial losses additionally depend on non-structural components - which are typically characterised by much higher failure rates - they are found to be less sensitive to the considered climate-change scenarios.
\end{abstract}

\section{Highlights}

- We compare potential effects of climate change on structural loads of OWTs.

- We assess structural loads on OWTs using an efficient surrogate modelling approach.

- We use performance metrics including damage, energy generation and financial losses.

- Fatigue damage was found more sensitive to climate change effects than energy density.

\section{Keywords}

Offshore wind energy; Climate change; Surrogate model; Financial loss

*Corresponding author details: david.wilkie.15@ucl.ac.uk 


\section{Word count}

7769 words

\section{List of abbreviations}

DLC - Design load case

FLS - Fatigue limit state

GP - Gaussian process

IEC - International electrotechnical commission

NREL - National renewables energy laboratory

OWT - Offshore wind turbine

OWF - Offshore wind farm

ULS - Ultimate limit state

\section{Introduction and motivations}

Offshore wind energy is becoming increasingly important as the proportion of energy generation form this source continues to grow, particularly in European countries. The International Energy Association forecasts that, under existing policy commitments, offshore wind energy production alone will represent up to $16 \%$ of electricity generation in the European Union by 2040 (1). An offshore wind farm (OWF) consists of a large number of offshore wind turbines (OWTs) distributed over a suitable area; for instance, the Hornsea One wind farm contains 1747 MW OWTs over an area of $407.34 \mathrm{~km}^{2}$ (2). OWT structures must be sufficiently robust to survive, and remain operational, throughout a long intended design life of 20-25 years (3). During this timeframe, OWTs are exposed to fluctuating loads which depend on site-specific environmental conditions in the form of wind and waves. In particular, wind acts as a driver of both structural loads and power production, by causing the blades to turn and drive a generator.

Recently, a probabilistic risk assessment framework for OWTs exposed to extreme wind and wave conditions (i.e., considering the ultimate limit state, or ULS) has been proposed by the authors, 1) enabling the modelling of these complex loads and their induced structural demands for use in financial loss calculations; and 2) considering an OWT as a system of interrelated structural and non-structural (e.g., equipment) components (4). The study presented here aims to extend this probabilistic risk assessment framework to also include operational wind and wave conditions and consider the fatigue limit state (FLS) which more often drives the design of OWT substructures in European waters (5). In this case, structural failure is due to the initiation and propagation of small cracks over the design life of an OWTs, which can grow to a critical size, threatening the integrity of 
the whole structure (6). Various OWT performance metrics such financial losses can also be computed through the proposed framework.

State-of-the-art approaches in designing and assessing OWTs (at both FLS and ULS) involve modelling environmental conditions using parametric probability distributions that are fit to historical environmental conditions measured at wind farm sites (e.g., (7)). Then, OWT structural demands are typically evaluated using advanced time-domain simulations representing OWT response to changing wind and wave conditions; however, these simulations can be computationally expensive. In fact, they explicitly account for the interaction between aerodynamic loads, hydrodynamic loads and structural response (commonly referred to as aero-hydro-servo-elastic simulation). In particular, load-induced fatigue damage is cumulative: it accounts for the full range of environmental conditions the OWT is exposed to, requiring an unfeasibly large number of time-history simulations (3). One remedy to use surrogate models (also known as metamodels or emulators), which replace the expensive time-history simulations with a statistical model fit to the results from a smaller number of intelligently chosen time-history simulations. In other words, surrogate models mimic the behaviour of the simulation model as closely as possible while remaining computationally cheaper to evaluate. Various surrogate modelling approaches have been recently proposed in the literature for OWTs on monopile foundations, including Gaussian Process (GP) regression (8-11) and for onshore wind turbines using Polynomial Chaos Expansion (12,13). These models significantly improve the computational efficiency of FLS assessment, and therefore also enable structural reliability assessment and the development of probabilistic risk models for OWTs to predict the potential financial impact of OWT failures. Such a probabilistic risk assessment framework can be further used (as proposed in this paper) to evaluate the potential impact of climate-change scenarios on the fatigue damage, structural safety and, ultimately, financial losses (cost of direct damage) of case-study OWTs.

Climate change is expected to affect weather patterns (14), with the potential to impact both OWT energy production and structural loading. However, quantifying the impact of climate change on environmental conditions is a challenging task. Warming of the poles will reduce the solar gradient across the globe, reducing mean wind speeds (and, consequently, wind energy production). However, various studies using climate change forecasting models have predicted localised increasing wind speeds in the North Sea, Baltic Sea and off the coast of Spain which are all feasible locations for future wind farms, as summarised by (14). Therefore, this study aims to also quantify the potential impact of climate-change scenarios on OWT power generation and resulting revenues.

The paper is organized as follows: Section 2 provides a brief overview of the impact climate change may have on the wind climate and how this might affect both OWT power production and OWT loading/fatigue damage. Section 3 introduces the proposed methodology for assessing site-specific structural loads and their induced damage using a GP regression to build a surrogate model for computationally expensive aero-elastic analysis. Following this, the proposed approach for estimating the financial impact of OWT fatigue failure in terms of direct damage is presented. The archetype OWT and the case-study site in 
Europe used in this paper are introduced in Section 4 together with the considered climate change scenarios. The results of this analysis are presented in Section 5 where potential climate-change-induced variations of financial losses are compared to changes in the predicted energy production as a result of climate change affecting wind speeds.

\section{Review of potential climate change impact on OWTs}

\subsection{Wind and wave climate}

The potential impact of climate change on renewable energy has been investigated by recent academic studies, summarised in a comprehensive literature review by Carvalho et al. (14). Each of the considered studies used general circulation models to project the consequence of changes in greenhouse gas emissions on the future climate. These projections are based on evaluating future environmental conditions in response to fixed assumptions about future emissions based on the emission pathways outlines in the Fifth Assessment Report of the Intergovernmental Panel on Climate Change; for example, Barstad, Soterberg and Mesquita (15) assume scenarios that result in an increase of global temperatures of $2{ }^{\circ} \mathrm{C}$ to $4{ }^{\circ} \mathrm{C}$ by 2100 .

With regard to wind, a north-south divide is predicted to develop within Europe, with increasing wind speeds, and therefore wind energy potential, in northern countries, and decreasing wind energy potential in the Mediterranean, for both onshore and offshore sites (14). This was concluded by reviewing studies that have focused on specific locations, including offshore sites in the Baltic Sea (15-17) and the Spanish Atlantic Coast $(15,18,19)$, predicting small increases in wind power density (i.e., the mean power available per square meter). The results for the North Sea are mixed, with both overall reductions predicted by Tobin et al. (17) but possible increases in the Northern North Sea $(15,16,19,20)$, in the Irish sea and along Ireland's Atlantic coastline (16). In all studies reviewed, the wind climate is represented by a Weibull distribution of 10-minute averages at a reference height of $10 \mathrm{~m}$ above sea-level. None of the studies reported on changes in turbulence intensity. A maximum increase in the mean wind speed distribution scale parameter of $0.05(\mathrm{~m} / \mathrm{s}) / 10$ year in the Baltic Sea and a maximum reduction of -0.05 on the North Coast of Spain were observed by Gaetani et al. (19); and the observed changes in the distribution shape parameter reported by this study were not statistically significant. These small changes in the scale parameter would result in a $1.5 \%$ change in the mean of the wind speed distribution at $10 \mathrm{~m}$ if extrapolated over a 30 -year period assuming the initial environmental conditions described in Section 4.2 of this paper. Similarly, Tobin et al. (17) reported changes in the Baltic mean wind speed between the periods 1971-2000 and 2071-2100, finding a $5 \%$ increase, i.e., a similar order of magnitude to the values reported by Gaetani et al. (19). In the North Sea, (20) estimated hub height mean wind speeds in the 2050's; using an ensemble of models, the study predicted a $\sim 3-4 \%$ average change in the annual mean wind speed over this period across all models and a $\sim 10-20 \%$ increase in the model with the largest change. Other results that predicted changes directly in terms of wind power density and not wind speed $(15,16)$ are not discussed. 
There are fewer studies focusing specifically on the wave climate; for instance, Carvalho et al. (14) found that the conclusion of studies into the impact of climate change on wave energy production were inconclusive. Hemer et al. (21) used an ensemble of previously developed models to evaluate changes in the wave climate. They reported small changes in wave heights (approximately $2 \%$ reductions in North Sea and Mediterranean, $4 \%$ increases on Spanish/Portuguese Atlantic coast) and spectral period ( 0 s to $0.2 \mathrm{~s}$ reductions).

\subsection{Impact on power production}

Wind energy power production $(P)$ is highly non-linear with respect to mean wind speed, as shown in Figure 1 for a National Renewable Energy Laboratory (NREL) 5MW archetype OWT (22). The ability of the OWT to extract energy from wind depends on OWT specific properties/components including the generator, torque control and other turbine control parameters (such as rotor speed and pitching). No power is produced at low wind speeds (when the mean wind speed is below the cut-in value) or at high wind speeds when the turbine is shut-down to protect the blades during more severe windstorms (when the mean wind speed is above the cut-out value). These changing operational states introduce additional non-linearity into the relationship between mean wind speed and power production. The calculation of this value is described in detail by Burton et al (23). For example, climate-change scenarios which increase the mean wind speed into the maximum power production range can cause the OWT to produce more electricity; however, this might be counteracted by the occurrence of stronger storms, forcing the OWT to stop rotating to protect its blades from damage at high wind speeds.

The yearly revenue $(R)$ resulting from operating an OWT can be calculated using the equation:

$R=P t_{a c t} C_{e}$,

where $t_{\text {act }}$ is the time in hours in which the power generating equipment is active over a year, and $C_{e}$ is the wholesale energy price for each unit of electricity generated. It should be noted that, in reality, the revenue will also be influenced by other complex factors such as turbine availability and grid capacity, and that the simplified calculation proposed in this paper is only used to compare different climate change scenarios for an individual OWT.

Existing studies have used climate-model projections to estimate the likely changes in weather given different climate-change scenarios, and then used wind energy density to compare the potential for power production. For instance, Barstad, Soteberg and Mesquita (15) predicted weak reductions (e.g., $0.5 \%$ in terms of estimated power production) in future wind-power potential over most of northern Europe during the next 30 to 40 years. 


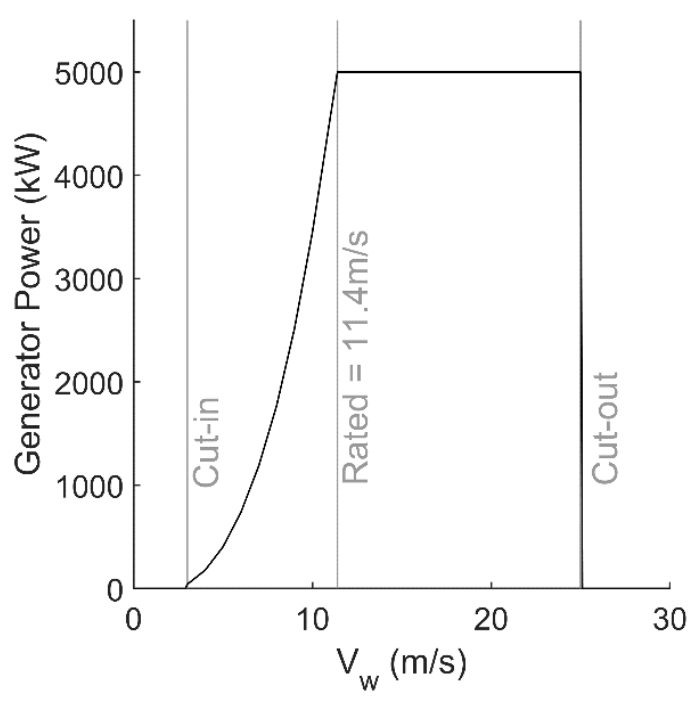

Figure 1: Power generated by a NREL 5 MW OWT.

\subsection{Impact on structural loads}

To date, no studies have assessed the impact of different climate change scenarios on OWT structural loads and resulting fatigue damage. However, one study focused on the West coast of India (24) highlighted that a 11-14\% increase in design wind speeds over the next 30 years is expected for OWTs installed in the Indian Sea. This time-scale is approximately equal to the operational life of an OWT if we assume an intended design life of 20-25 years with $25 \%$ life extension (25).

Structural wind loads on OWT are driven by aero-dynamic drag and lift. For example the aero-dynamic loading per unit length at time " $\mathrm{t}$ " $\left(f_{a}(t)\right)$ on the tower is calculated as a function of the square of the instantaneous wind speed $(V(t))(26)$ :

$f_{a}(\mathrm{t})=0.5 C_{d} G_{f} \rho A V(t)^{2}$,

where $A$ is the cross-sectional area of the structure, $C_{d}$ is the drag coefficient, and $G_{f}$ is a gust factor (that accounts for the effect of wind actions because the peaks in wind pressure may not occur simultaneously with structural vibrations (26)). In OWT load assessment, blade loading is typically calculated using different lift and drag equations which are similar in form to Eq.(2) (23), but written as differential equations along the blades length and solved using finite element analysis. The resulting systems of equations to evaluate structural response are coupled together to predict dynamics of the full OWT structure, e.g., (27).

The structural design of OWTs in European waters is often driven by the FLS (5). This limit state addresses cracks which grow as a result of stress cycles in the OWT structural components which in turn result from stochastic external loading (in the form of wind and waves) and the cyclic motion induced by the rotation of the rotor. Current industry practice is to estimate the 
impact of crack growth during the design stage using the SN curve approach (28), an example of which is shown in Figure 2. This assumes that the damage caused by each stress cycle over the design life of an OWT can be summed (i.e., it is linear). The damage per stress cycle is calculated as the ratio of the number of cycles occurring at a given stress level (over the structures design life) divided by the tolerable number of cycles the structure could withstand at that stress level before a through wall thickness crack forming, as defined by experimental tests (28). The tolerable number of cycles $\left(N_{j}\right)$ can be predicted using the equation:

$N_{j}=K \Delta S_{j}^{m}$,

where $\Delta S_{j}$ is the applied stress range, $K$ is the intersection of the SN curve with the number of cycles axis (Figure 2), $m$ is the slope of the SN curve; the index refers to the $j^{\text {th }}$ cycle in a stress time history. The SN curve slope depends on the material being assessed; the gradient of the SN curve typically ranges between three or 10 (29). As a result, fatigue loads depend on wind speed values raised to a power of $2+m$ and, therefore, are sensitive to changes in the wind climate. In addition, OWTs experience wave loading which itself depends on wind speed, as discussed in Section 3.1.

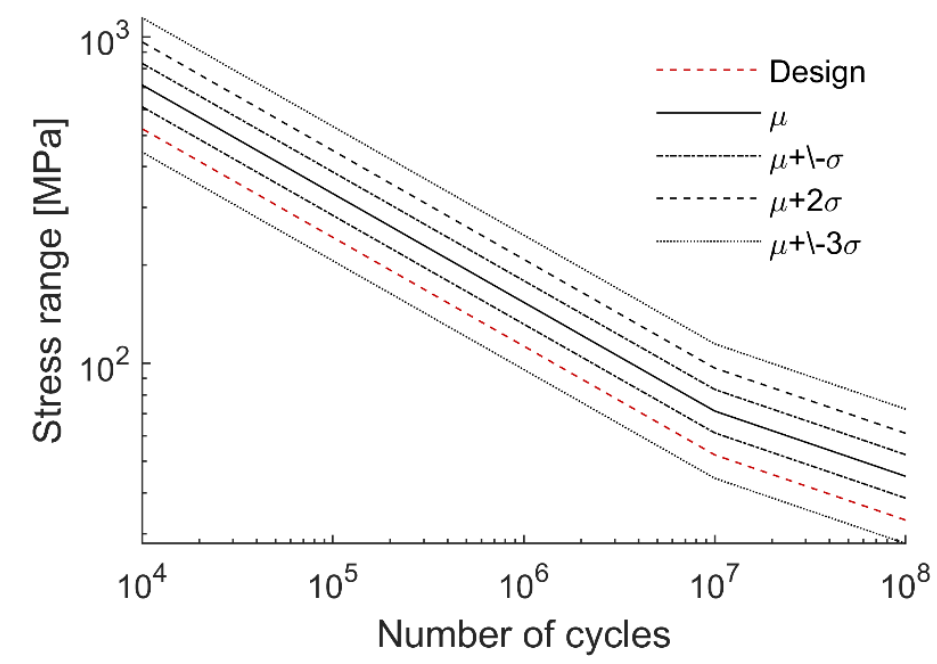

Figure 2: SN curve from DNVGL (28) for a butt weld; the figure shows the scatter in the SN-curve when the full distribution of $x$ axis intersections is utilised. The $2.5^{\text {th }}$ percentile curve is generally used in structural design and is highlighted as the mean curve minus two standard deviations.

\section{Loss assessment methodology}

The impact of changes in the environmental conditions on OWT loading can be quantified through changes in the rate of structural failure of the OWT components. If information about the failure of the component is known, failure rates can be converted into financial losses, providing stakeholders with more widely interpretable metrics for decision making. 
Furthermore, structural failure rates due to the FLS can be combined with failure rates of the other non-structural components (such as the generator or blades) using the loss calculation procedure described in the following sections. Specifically, this calculation consists of three distinct components, as shown in Figure 3. The individual steps are:

- Simulation-based assessment of the FLS damage for the OWT structural components (i.e., tower and monopile). To this end, a surrogate model is built to represent the fatigue damage at different combinations of the environmental conditions on a small sample of these conditions.

- Computation of the FLS failure rates for OWT structural components over their design life, by drawing a larger second sample of fatigue damage predictions from the surrogate model, which is efficient to evaluate. These samples follow the joint probability distribution of the environmental parameters, described in Section 3.1, which can be updated to reflect the assumed climate-change scenarios as described in Section 4.3. Additional uncertainties (such as those modelling the variability in fatigue material properties) can be included in the calculation.

- Estimate financial loss by combining the computed failure rates of OWT structural components with non-structural components using published empirical data, e.g., failure rate and cost; (30).

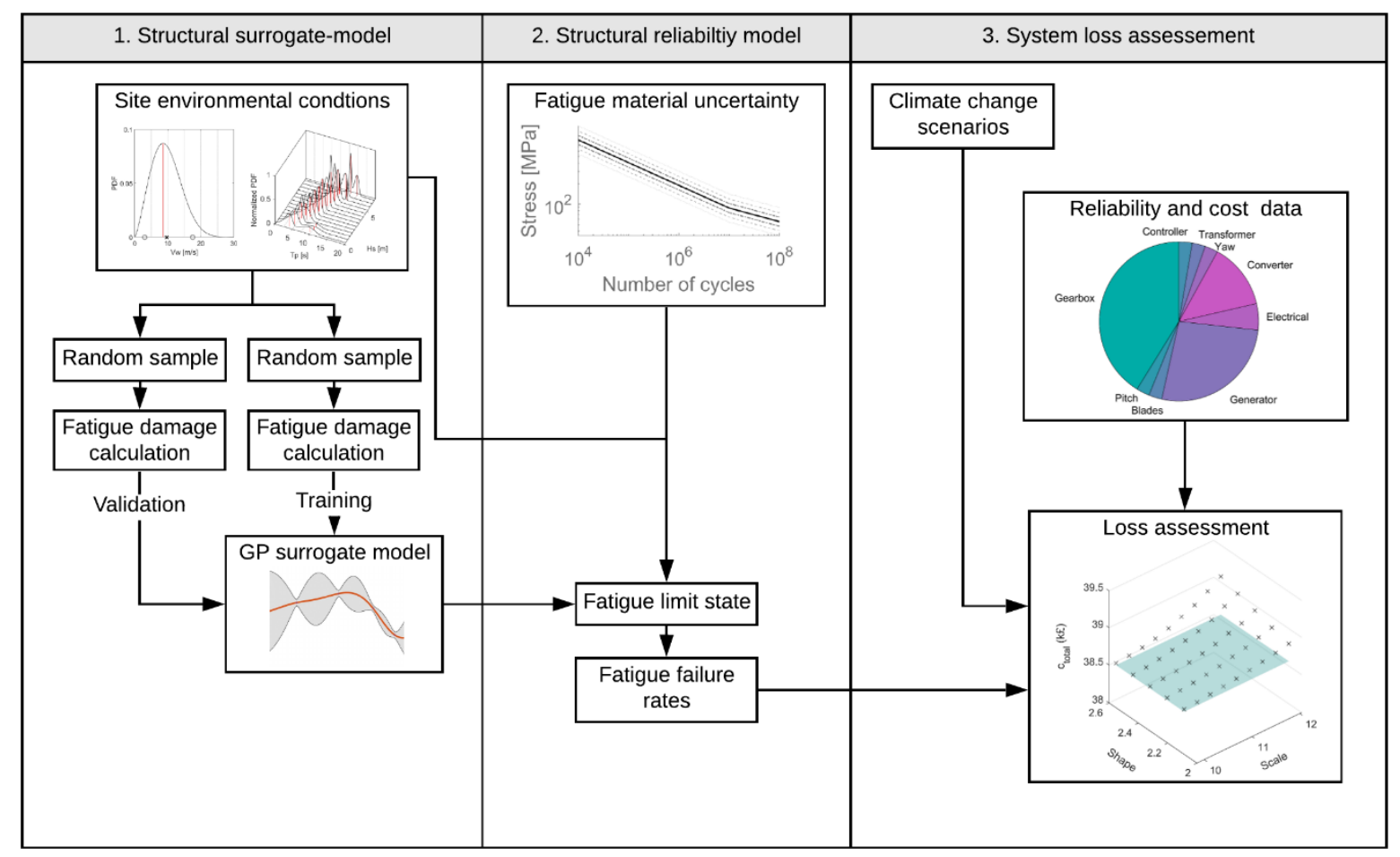

Figure 3: Proposed OWT loss assessment framework considering both structural (for FLS) and non-structural components. 


\subsection{Fatigue damage assessment}

Structural loads acting on OWTs are assessed according to the International Electrotechnical Commission (IEC) code 614003. This document defines a series of different design load cases (DLCs) representing different conditions an OWT may experience during its intended design life. It includes various loading conditions for the FLS where the OWT is operational (e.g., DLC 1.2) or where the turbine is shut-down due to high wind speed (e.g., DLC 6.4). A range of other DLCs are specified to cover fault conditions, transient start-up and shut-down conditions.

Fatigue damage is cumulative; therefore, the DLCs for the FLS require multiple structural simulations performed across all the relevant wind and wave conditions. These are defined in terms of all the important variables affecting the wind and wave climate distributions, and typically include: mean wind speed $\left(V_{w}\right)$, turbulence intensity $\left(T_{i}\right)$, wind direction $\left(\theta_{w i}\right)$, significant wave height $\left(H_{s}\right)$, wave peak spectral period $\left(T_{p}\right)$ and wave direction $\left(\theta_{w a}\right)$. Simulation inputs are determined by drawing random samples from the joint/conditional probability distributions which define the occurrence of these variables. In the IEC 61400-3, the assessment can be simplified by allowing $V_{w}$ to be discretised into $2 \mathrm{~m} / \mathrm{s}$ wide bins and considering a single value of $H_{s}$ for each bin. Nevertheless, a higher accuracy approach, consisting of many more samples, is necessary to accurately predict fatigue damage (5). The fatigue damage calculation follows the outline provided in Section 2.3; however, more details of the specific assumptions used in this study are provided here and the overall calculation approach is described in Figure 4.

Firstly, specific values of the considered environmental variables are selected and a time-domain aero-elastic simulation is run for each combination of those variables using the aeroelastic computer-aided engineering software OpenFAST (27). Within OpenFAST, turbulent wind histories are generated using TurbSim (31) and irregular wave time series are generated using HydroDyn (32). From these time series, aero-dynamic loads, are calculated as described in Section 2.3 and hydro-dynamic loads are calculated using Morrison's equation (33) which depends on the water particle displacement $(H(t))$ and velocity $(\dot{H}(t))$. These calculations are conducted for each component of the turbine and require specification of relevant drag and lift factors for the cross-sectional properties of the structural component, which for a NREL 5MW OWT are provided by Jonkman et al. (22).

The output is a time series of loads, for example at the base of the monopile, which are then converted into component stress time histories. These are processed using the rainflow-counting algorithm (34) to extract the stress ranges occurring during the time series. For each simulation, a single value of fatigue damage is predicted by using an SN curve (Eq.(3)), by 1) determining the number of tolerable cycles at each stress range; then 2) dividing the observed number of cycles $\left(n_{j}\right)$ by the number of tolerable cycles and summing over each stress cycle in the time history. The fatigue damage over the OWT design life can be calculated by linearly scaling the obtained damage value from the time-history structural simulation length to the assumed design life of the OWT. Additionally, due to the stochastic nature of the generated wind and wave time series, each analysis 
has to be repeated for every set of environmental conditions in order to produce a stable estimation of the total fatigue damage (where the analysis repetitions are referred to as "seeds").

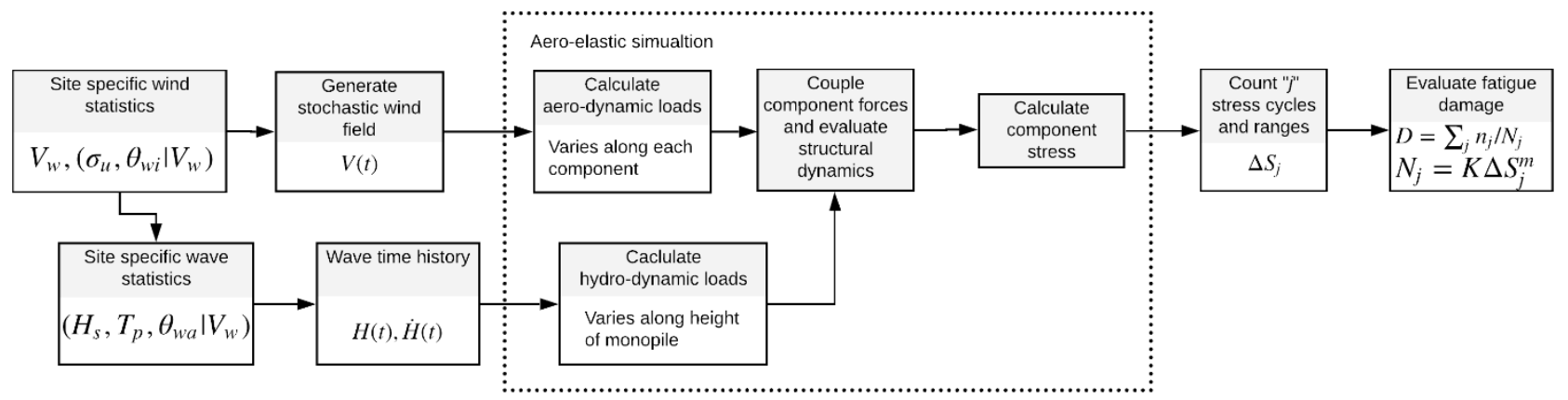

Figure 4: Flowchart of the fatigue damage calculation from the parameters used to describe the environmental conditions to fatigue damage. Where $f($.$) refers to some functional relationship described in the main text.$

The total fatigue damage over the life of the structure can be calculated by averaging the predictions of fatigue lifetime:

$D_{\text {total }}=\frac{1}{N_{\text {sim }}} \sum_{j=1}^{N_{\text {sim }}} D_{j, l i f e}$

where $D_{\text {total }}$ is the total predicted damage for the OWT at the site, $N_{\text {sim }}$ is the number of damage samples (i.e., the product of the number of samples of environmental conditions and number of seeds) which are indexed by $j, D_{j, l i f e}$ is the lifetime fatigue load predicted for each sample of the environmental conditions.

Finally, a limit state (or performance) equation is introduced to model OWT structural component failure as a function of the total damage and random variables related to the maximum tolerable fatigue damage $\left(X_{\delta}\right)$ and the uncertainty in the SN curve $\left(X_{S N}\right)$; the characterisation of these variables is presented in Table 1:

$G\left(X_{\delta}, X_{S N}\right)=X_{\delta}-X_{S N} D_{t o t a l}$.

The limit state depends on two random variables defined by the probability distributions shown in Table 1, although it should be noted that a larger set of random variables can be considered to capture other modelling uncertainties e.g., (11). Failure of the structure/structural component is assumed to occur when the limit state equation assumes negative values, and the probability of failure can be computed as $\operatorname{Pr}(G \leq 0)$. This can be solved numerically 1$)$ by drawing samples from all the random variables involved in the assessment; 2) by evaluating the limit state equation for each set of samples; and 3) by averaging over the number of observed failures. This enables calculation of the probability of FLS failure for a given prediction of lifetime damage by averaging over the number of observed failures. In fact, OWT structures are normally designed to obtain very low probabilities of failure, which implies that the limit state equation must be solved several thousand times, resulting in a prohibitively large computational effort. Full details on the proposed simulation-based structural reliability assessment 
can found in (11). An accurate technique for problems with a low number of random variables is importance sampling (35) and is used in this study, as further described in Section 5.3.

Table 1: Statistical properties of the random variables in limit state equation Eq.(5) (11).

\begin{tabular}{lccc}
\hline Random variable & Distribution & Mean of logarithm & $\begin{array}{c}\text { Standard deviation of } \\
\text { logarithm }\end{array}$ \\
\hline Tolerable damage $\left(X_{\delta}\right)$ & Log-normal & 0 & 0.3 \\
SN Uncertainty $\left(X_{S N}\right)$ & Log-normal & -0.9 & 0.46 \\
\hline
\end{tabular}

\subsection{Surrogate model development}

The main challenge in performing the assessment described in the previous section is that large computational resources are required to obtain an accurate estimation of lifetime fatigue damage. In fact, dynamic structural simulations must be run for many stochastic wind and wave loading conditions. Surrogate modelling represents a potential solution to this challenge, allowing one to replace the expensive computer-based simulation model - which links environmental conditions to fatigue damage - with a simpler statistical model. A surrogate model is generally trained using a much smaller number of aero-elastic simulations than required for a full fatigue damage assessment; this training sample is selected using an appropriate design of experiment technique (36). Specifically, a GP regression is used in this study to build a surrogate model for lifetime fatigue damage, primarily because of its ease of application and its demonstrated suitability for applications concerning OWTs on monopile foundations in the existing literature $(8,9,37)$.

GP-regression predictions consist of a mean component, modelling the overall trends in the training data, and a GP constructed through the residuals to interpolate between training samples based on the assumption that there is a spatial correlation between the model predictions. To this aim, the GP is characterized by a covariance function which is selected in advance. Most of the widely used covariance functions in the literature depend on the distance between input samples, capturing the fact that two samples of the input variables (e.g., mean wind speed in this study) which are close are expected to produce similar model outputs (i.e., fatigue damage in this study). GP predictions require all the data points they are trained upon, meaning that large sample sizes can still be computationally expensive. A comprehensive description of the background theory of GP regression and discussions on underlying hypotheses is provided by Santner, Williams and Notz (36).

In this study, a training sample set is generated by randomly sampling from the probability distributions describing the climate conditions, described in Section 4.2. This design of experiment procedure was selected because, as it can be seen from Eq.(4), lifetime fatigue damage prediction is a problem of mean estimation: a random sampling scheme is expected to perform well in this context (36). This study follows the recommendations in Wilkie and Galasso (11) and uses 500 samples and 20 seeds. A larger number of seeds than strictly necessary were used because of available high-performance computing resources. The GP regression used in this study has a constant mean function and uses a Matern 5/2 covariance function (38). This model is 
fit to the training data using the maximum likelihood estimation approach. The error in the model prediction is evaluated using a leave-one-out cross-validation approach where the error is sequentially evaluated for a GP regression fit by using each sample in the training sample set except one.

\subsection{Loss computation}

A simplified loss assessment procedure is proposed to combine failure rates for the OWT structural components with those recorded empirically for the non-structural components (e.g., equipment). The procedure is only sketched in brief as full details are provided in Wilkie and Galasso (4). It consists of generating different failure scenarios from all possible combinations of different states in a system with a given number of components. For example, if a system of $N$ components has two failure states (i.e., failure or operation), there will be $2^{N}$ combinations. This is achieved by defining a logical matrix of failure scenarios $(\boldsymbol{K})$ with rows equal to the number of components, and columns equal to the number of failure states. A financial cost can be associated to each failure scenario, by multiplying $\boldsymbol{K}$ by the component failure costs, resulting in the cost matrix $\boldsymbol{K}_{\boldsymbol{c}}$. The probability of incurring a set $\operatorname{cost}\left(P_{s y s}\left(c_{r}\right)\right)$ can then be estimated as a sum over the subset of $\boldsymbol{K}_{\boldsymbol{c}}$ which has the total cost $c_{r}$ denoted $\boldsymbol{k}^{*}$ :

$P_{s y s}\left(c_{r}\right)=\sum_{\boldsymbol{k}^{*} \in \boldsymbol{K}_{\boldsymbol{c}}} \prod_{i=1}^{N} P_{i}^{k_{i}}\left(1-P_{i}\right)^{1-k_{i}}$

where $P_{i}$ is the probability of failure of the $\mathrm{i}^{\text {th }}$ component, $k_{i}$ is a logical value indicating whether or not the $\mathrm{i}^{\text {th }}$ component fails during the failure scenario and $c_{r}$ is a pre-selected cost level.

The expected loss $\left(L_{\text {total }}\right)$ can then be computed as an expectation over different cost levels:

$L_{\text {total }}=\sum_{c_{r}} P_{\text {sys }}\left(c_{r}\right) \cdot c_{r}$.

The equipment failure and cost data used in this study is taken from the work of Carroll, McDonald and McMillian (30); the study surveyed failure rates for OWT components from databases of observed failures, as summarized on Table 2. The structural probability of failure is calculated following the methodology outlined in Section 3.2 and is converted into a failure rate by assuming that failures follow a Poisson distribution.

Table 2: Main non-structural component failure rates and costs for a case-study OWT (30).

\begin{tabular}{lcc}
\hline \hline Component & Major replacement $(\boldsymbol{\epsilon})$ & $\begin{array}{c}\text { Failure rate } \\
(/ \text { turbine/year) }\end{array}$ \\
\hline Gearbox & 230,000 & 0.154 \\
Hub & 95,000 & 0.001 \\
Blades (per blade) & 90,000 & 0.001 \\
Transformer & 70,000 & 0.001
\end{tabular}




\begin{tabular}{lll} 
Generator & 60,000 & 0.095 \\
Circuit breaker & 14,000 & 0.002 \\
Power supply & 13,000 & 0.005 \\
Pitch system & 14,000 & 0.001 \\
Yaw system & 13,000 & 0.001 \\
Controller & 13,000 & 0.001 \\
\hline
\end{tabular}

\subsubsection{Monopile cost}

The cost of the monopile $\left(c_{\text {mon }}\right)$ is estimated using the equation proposed by (39) in Euros:

$c_{\text {mon }}=320 P_{W T}\left(1+0.02\left(h_{\text {water }}-8\right)\right)\left(1+1 \cdot 10^{-7}\left(h_{\text {hub }}\left(0.5 \phi_{R}\right)-10^{5}\right)\right)$.

where the cost estimate depends on the rated capacity $\left(P_{W T}\right.$, in MW), the water depth $\left(h_{w a t e r}\right.$, in $\left.\mathrm{m}\right)$, the hub height above the mean sea level $\left(h_{h u b}\right.$, in $\left.\mathrm{m}\right)$, and the rotor diameter $\left(\phi_{R}\right.$, in $\left.\mathrm{m}\right)$. The equation was adapted from a 2003 study and was validated against actual foundation costs from five real OWFs. The average error was approximately $8.7 \%$, but Eq.(8) outperformed two other cost models, particularly in terms of foundation cost.

\section{Illustrative application}

\subsection{Considered OWT}

The OWT considered in this study is a NREL 5MW reference turbine (22) with a monopile foundation, which is shown in Figure 5 and has the properties listed in Table 3 . The turbine has a cut-in speed of $3 \mathrm{~m} / \mathrm{s}$ and a cut-off speed of $25 \mathrm{~m} / \mathrm{s}$ : when this mean wind speed is exceeded, the rotor enters its parked state by pitching the blades into the wind to prevent damage. The rated wind speed is $11.4 \mathrm{~m} / \mathrm{s}$, after which the controller starts to pitch the blades out of the incoming wind flow to regulate the rotational speed of the generator. The hub is supported by the tower and is located at an Elevation (EL) of $+87.6 \mathrm{~m}$ above the mean sea level and is attached to a $126 \mathrm{~m}$ diameter 3-bladed rotor. The monopile support structure spans from EL $+10 \mathrm{~m}$ to the mudline at EL - $20 \mathrm{~m}$. At the base of the tower, a transition piece connects the tower and monopile. A full list of dimensions and material properties of the turbine structure are provided by Jonkman et al. (22). The analysis length is set to 11 minutes, allowing the first minute to be cut (which includes transient loads due to the simulation initial conditions) while leaving the full 10-minute analysis length. The SN curve used in this study is shown on Figure 2 and is taken from DNVGL-RP-C203 (28) for a circumferential butt weld from both sides, referred to as type $\mathrm{D}$ in the code.

The foundation is modelled using an apparent fixity model, which represents foundation flexibility by extending the monopile below the mudline. The extension length is chosen to match the natural frequency from a spectral analysis where the monopile is embedded in a linear-elastic soil matrix. This assumes an embedded pile length of $30 \mathrm{~m}$ using soil properties from Damgaard et al. (40). 


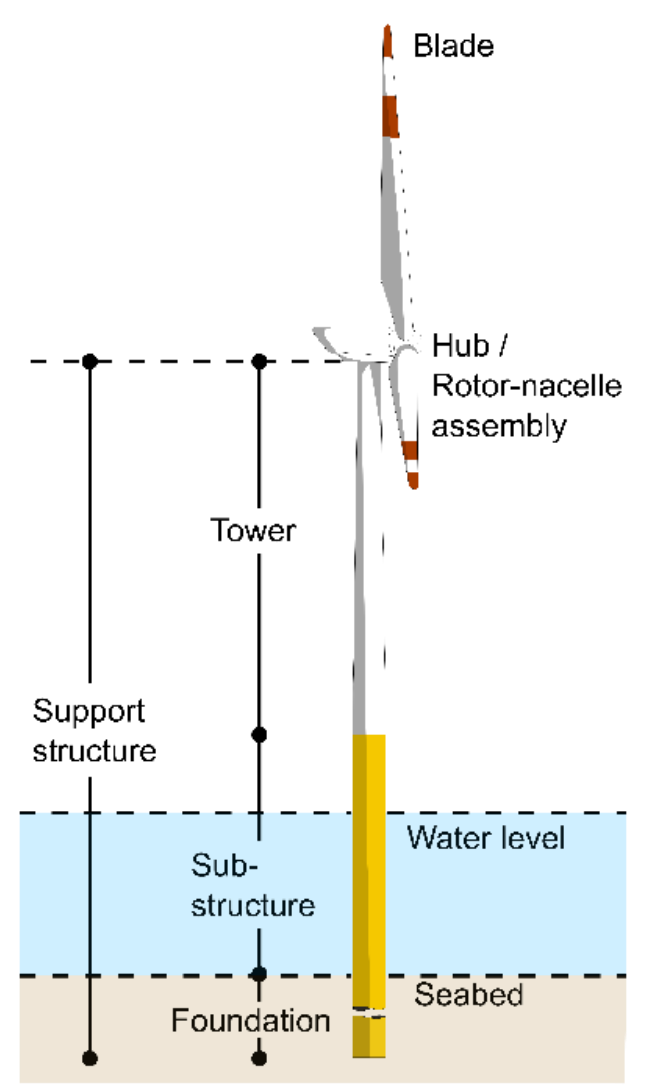

Figure 5: Schematic of the NREL 5MW OWT.

Table 3: Main dimensions for the NREL 5MW OWT.

\begin{tabular}{ll}
\hline \hline Parameter & Value \\
\hline Mass of the rotor-nacelle assembly $(\mathrm{kg})$ & 350,000 \\
Tower diameter bottom, top $(\mathrm{m})$ & $6,3.87$ \\
Tower wall thickness bottom, top $(\mathrm{mm})$ & 35,25 \\
Monopile diameter $(\mathrm{m})$ & 6 \\
Monopile wall thickness $(\mathrm{mm})$ & 60 \\
\hline
\end{tabular}

\subsection{Site environmental conditions}

This study uses environmental data measured by the FINO3 met-mast located in the German North Sea, which is part of the FINO project (41). The site has 7-years' worth of continuously recorded environmental data and the site depth varies between $22 \mathrm{~m}$ and $30 \mathrm{~m}$ ). This raw data was processed into probability distributions by Hübler, Gebhardt and Roles (42) who discretized the data into bins of different mean wind speeds, which is the only independent distribution. The mean wind speed distribution follows a Weibull distribution, which has a probability density function (PDF): 
$f\left(V_{w}\right)=\frac{k}{\lambda}\left(\frac{V_{w}}{\lambda}\right)^{k-1} e^{-(x / \lambda)^{k}}$

with a scale parameter $(\lambda)$ of $10.94 \mathrm{~m} / \mathrm{s}$ and a shape parameter $(k) 2.32$. A series of conditional distributions were determined for the other environmental conditions including turbulence intensity, significant wave height, peak spectral period, wind angle and wave angle. These distributions were randomly sampled 500 times in order to generate input environmental conditions (for training the surrogate model), at which to evaluate the considered NREL 5MW OWT using OpenFAST, as shown on Figure 6. Full details of the distributions and parameters used in this study and are provided in the appendices of Hübler, Gebhardt and Roles (42); however, a summary of the relationship between the different variables and the distributions used are presented on Table 4 .
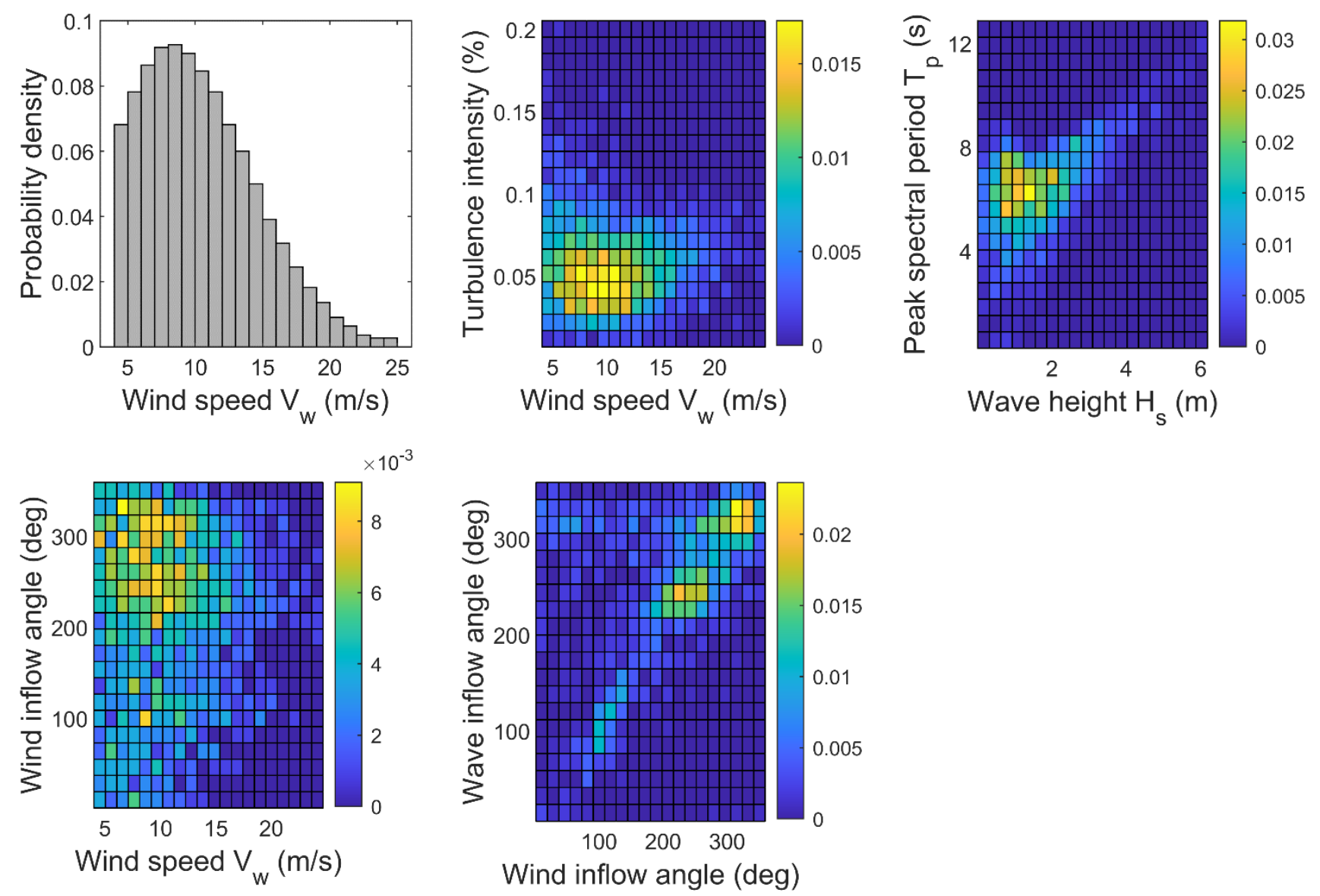

Figure 6: Random samples drawn from the FINO3 site climate distributions and used to fit the GP regression model.

Table 4: Probability distributions used to model environmental conditions at the FINO3 site showing the conditional dependencies, the distribution parameters are defined by Hubler et al. [REF]. * Kernel Density Estimation 


\begin{tabular}{lclc}
\hline \hline \multicolumn{1}{c}{ Parameter } & Variable & \multicolumn{1}{c}{ Distribution } & Dependencies \\
\hline Mean wind speed & $V_{w}$ & Weibull & N/A \\
Turbulence intensity & $T_{i}$ & Weibull; Gamma & $V_{w}$ \\
Significant wave height & $H_{s}$ & Gumbel; Weibull & $V_{w}$ \\
Peak spectral period & $T_{p}$ & Bimodal Gumbel & $H_{s}$ \\
Wind direction & $\theta_{\text {wind }}$ & Non-parametric $\mathrm{KDE}^{*}$ & $V_{w}$ \\
Wave direction & $\theta_{\text {wave }}$ & Non-parametric $\mathrm{KDE}^{*}$ & $H_{s} ; \theta_{\text {wind }}$ \\
\hline
\end{tabular}

\subsection{Climate-change modified environmental conditions}

Based on the literature review in Section 2.1, climate change may have different effects on the mean wind speed experienced by an OWT depending on its location (e.g., if the site is located in the North or South of Europe). Due to the lack of clear trends (as discussed above), this paper takes a parametric approach and focuses only on the mean wind speed as small changes in the other parameters were found in Section 2.1. It should be noted that the marginal distribution of the other environmental parameters will change as a result of their conditional dependence on the mean wind speed described in Table 4.

The distribution of the mean wind speed was varied in order to evaluate the consequence for fatigue damage and power production, using the two parameters defined in Eq.(9). Specifically, a $\pm 14 \%$ change in the scale parameter was selected to cover the maximum range found by the predictions of Devis, Van Lipzig and Demuzere (20) which were evaluated over a 30 year period; while a $\pm 7 \%$ change in the shape parameters was selected for illustrative purpose, although small variations of this parameter were predicted by Gaetani et al. (19). These ranges are sampled with nine and five uniformly spaced points for the scale and shape parameter respectively, and 10,000 samples for each combination. The parameters of the other conditional distributions (i.e., turbulence intensity, significant wave height, peak spectral period, wind inflow angle and wave inflow angle) remain the same. However, because of their conditional dependence on the mean wind speed, the marginal distributions of the other parameters will change when the mean wind speed distribution is updated. For example, when the mean wind speed distribution is updated with the extreme scale parameter samples, the mean of the significant wave height changes by $+/-12$ $\%$. However, the shape parameter was found to have a very small influence on the distributions of the other variables.

The effects of changing the distribution parameters for the mean wind speed is shown in Figure 7. The resulting variations in terms of the mean of the distribution is shown in Figure 7 (left); it is observed that the shape parameter has very little impact as the equation for the Weibull mean $\left(\mu_{w e i}\right)$ is $\mu_{w e i}=\lambda \Gamma(1+1 / k)$, and the gamma function $(\Gamma()$.$) is flat over the region near$ $k=2.32$. Figure 7 (middle) shows that the probability of the turbine being non-operational due to low mean wind speeds 
(below $3 \mathrm{~m} / \mathrm{s}$ ) depends on both the scale and shape parameter. A similar result is shown in Figure 7 (right) for the probability of high wind speeds (above the $25 \mathrm{~m} / \mathrm{s}$ cut-off), where much lower values than in Figure 7 (middle) are observed because of the low mean of the wind speed distribution (Figure 7 (left)) compared to the cut-off wind speed. The occurrence of these nonoperational conditions is important as the wind turbine will not produce electricity while they persist.
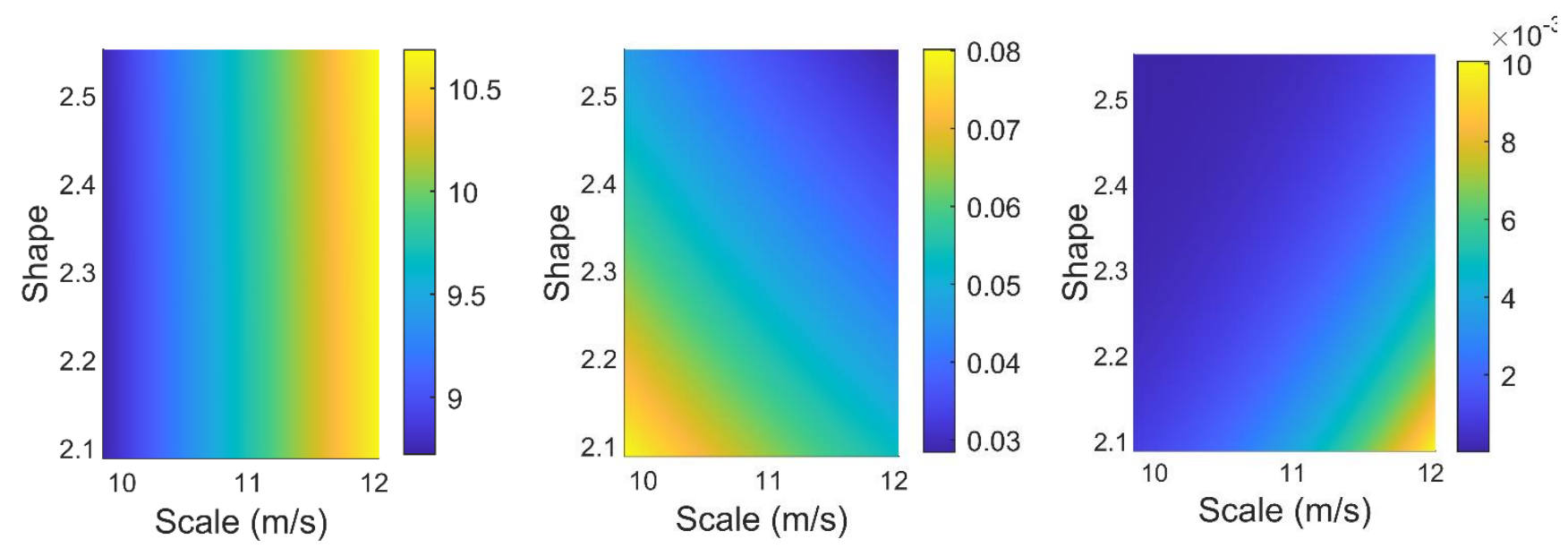

Figure 7: Impact of scale and shape parameters on the mean of the wind speed distribution (left); proportion of 10-minute wind speed less than start-up wind speed (middle); and proportion of 10-minute wind speed greater than the cut-out wind speed (right).

\section{Results and discussion}

\subsection{Surrogate model}

The mean surrogate-model predictions for lifetime fatigue damage are shown in Figure 8 for different combinations of the environmental variables; a contour plot of the environmental samples used to fit the surrogate model is also shown below each surface, to indicate where the training samples were located. The accuracy of the GP-regression model was assessed using the normalised mean squared error (NMSE) on the leave-one-out cross-validation set; this is calculated:

$N M S E=1-\frac{\sum\left(D_{j, l i f e}-G P\left(\boldsymbol{x}_{j}\right)\right)^{2}}{\sum\left(D_{j, \text { life }}-\text { mean }\left(\boldsymbol{D}_{\text {life }}\right)\right)^{2}}$

where $G P\left(\boldsymbol{x}_{\boldsymbol{j}}\right)$ is the GP prediction of damage for the $j$-th training environmental sample $\boldsymbol{x}_{\boldsymbol{j}}$ and $D_{j, l i f e}$ is the value of lifetime fatigue damage predicted by OpenFAST for the $j$-th sample. The error was found to be 0.962 , where 1 would indicate the GP explains the variance in the data perfectly, i.e., an error of less than $4 \%$ was found.

Figure 8 (left) shows that the peak in fatigue damage occurs around the rated mean wind speed $(11.4 \mathrm{~m} / \mathrm{s})$, as also identified by Zwick and Muskulus (43). The increase in fatigue damage visible in Figure 8 (middle) occurs around a $T_{p}$ of $4 \mathrm{~s}$, at which the peak in the wave loading spectrum approaches the fore-aft natural frequency of the OWT and consequently causes larger loading. The samples of misalignment angle are grouped around $0^{\circ}$ in Figure 8 (right) because wind and wave inflow were 
generally found to be aligned and the misalignment angle was calculated as the absolute value of the wind inflow angle minus the wave inflow angle.

Using the FINO measured environmental conditions, the overall damage was calculated to be 0.11 by solving Eq.(4) using Monte Carlo simulation with 10,000 samples; similarly the average power production was calculated to be $2826.72 \mathrm{~kW}$.
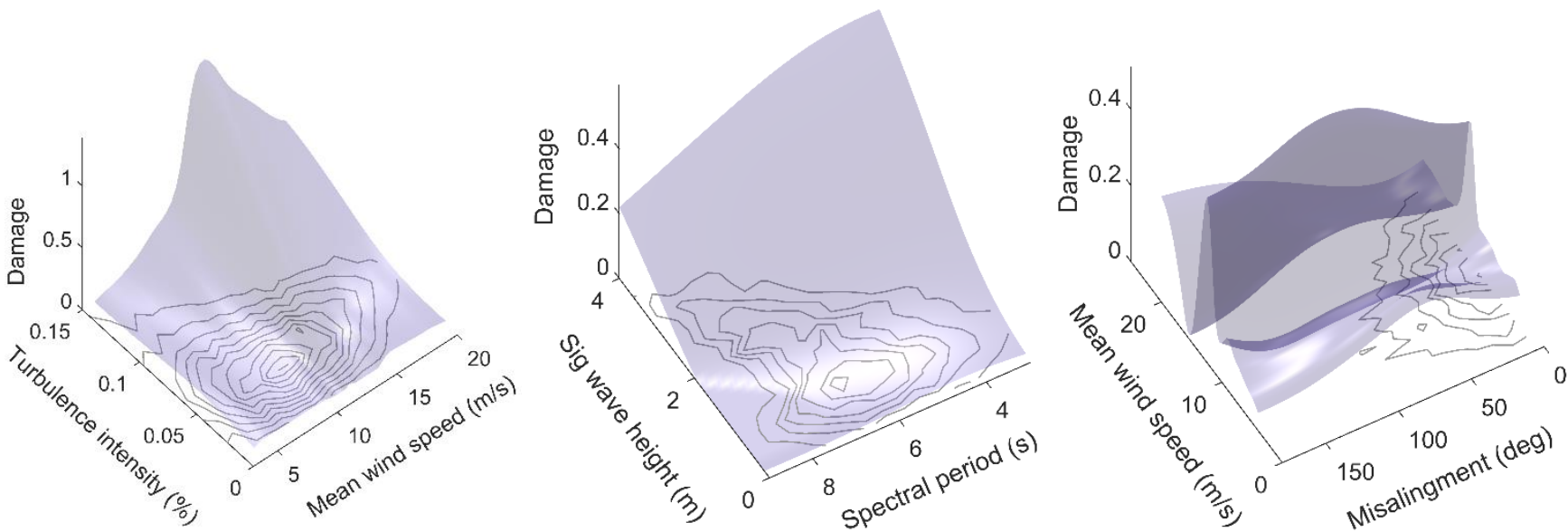

Figure 8: GP-regressions predictions of fatigue damage as a function of different environmental variables and contour plots of the environmental conditions in the sample used to fit the GP. Turbulence intensity and mean wind speed with $H_{s}=1.5 \mathrm{~m}, T_{p}=7 \mathrm{~s}, \theta_{m i s}=0^{\circ}$ (left). Significant wave height and peak spectral period with $V_{w}=9 \mathrm{~m} / \mathrm{s}, T_{i}=0.05, \theta_{m i s}=0^{\circ}$ (middle). Mean wind speed and wind and wave misalignment with $T_{i}=0.05, H_{s}=1.5 \mathrm{~m}, T_{p}=6 \mathrm{~s}$ (right).

\subsection{Fatigue damage and power at changing environmental conditions}

The fatigue damage and average power production are dependent on the wind speed distribution parameters (see Figure 9), where the scale parameter has a greater influence than the shape parameter in both cases. The average power production was calculated by taking the average of the power production values listed in Figure 1 over all samples of the environmental conditions (equivalent to averaging over the power predicted by each time-history analysis). The parameters of the quadratic surface shown in Figure 9 (left) were calibrated using least squares fitting to describe a continuous function of the average power generation $(\mathrm{P}(\lambda, k))$ across all the data-points with a high adjusted $\mathrm{R}$-squared value of 0.992 :

$\mathrm{P}(\lambda, k)=858.4+365.4 \lambda-1807.3 k+192.4 \lambda k-23.6 \lambda^{2}+13.8 k^{2}$.

Figure 9 (left) shows that the average power generation increases as the scale parameter increases (note that the scale parameter has units of $\mathrm{m} / \mathrm{s}$; therefore, the units of the fitting coefficients in Eq.(11) and Eq.(12) are consistent with each equation output and can be easily obtained through dimensional analysis). This behaviour is expected from Figure 7 (left) as power production is directly affected by the mean wind speed. Additionally, increasing the scale parameter reduces the proportion of samples falling below the cut-in (Figure 7 (middle)). However, it also increases the proportion of samples above the cut-out, but this has a limited effect on average power production as mean wind speeds above $25 \mathrm{~m} / \mathrm{s}$ are rare anyway (Figure 7 (right)). 
Fatigue damage against the scale and shape parameters are shown in Figure 9 (right), where the damage surface $(D(\lambda, k))$ is modelled using a quadratic relationship with a high adjusted R-squared value of 0.969 :

$D(\lambda, k)=0.080+0.012 \lambda-0.121 k+0.015 \lambda k-1.41 \cdot 10^{-3} \lambda^{2}-6.90 \cdot 10^{-3} k^{2}$.

The fatigue damage is found to increase at a faster rate than the average power generation. When the shape parameter is held at its original value (2.32) and the scale parameter is increased to its maximum value, the average power generation increases from $2,799 \mathrm{~kW}$ to a maximum of $3,096 \mathrm{~kW}$, a $10 \%$ increase. When the scale parameter is reduced to its minimum value, the average power generation reduces from $2,799 \mathrm{~kW}$ to a minimum of $2,448 \mathrm{~kW}$, a $14.33 \%$ reduction. In contrast, when the scale parameter is increased to its maximum value, fatigue damage increases from an original value of 0.108 to maximum of 0.124 , a $13 \%$ increase, and reduces from 0.108 to a minimum 0.089 , a $17 \%$ reduction when the scale parameter is reduced to its minimum value. In terms of shape parameter, the sensitivity of both average power generation and fatigue damage is much lower. In particular, when the shape parameter is changed and the scale is held at its original value of 10.94, the average power generation reduces from a maximum of $2,339 \mathrm{~kW}$ to $2,716 \mathrm{~kW}$ an $\sim 6 \%$ change; whereas, the damage reduces from a maximum of 0.111 to a minimum of 0.105 , a $5 \%$ reduction.
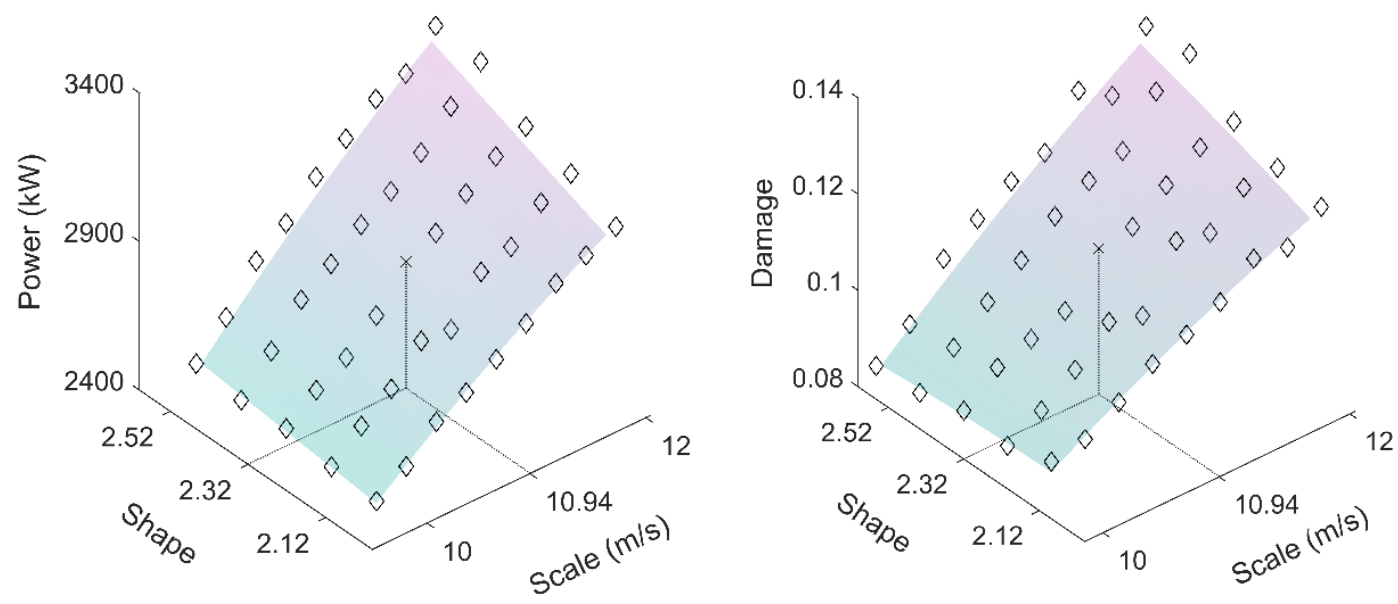

Figure 9: Predicted average power production (left) and fatigue damage (right) for various values of the scale and shape parameters of the mean wind speed distribution. In both the nominal power and damage have been highlighted with an ' $x$ '.

\subsection{Comparison between revenue and financial losses}

There is a linear relationship between electricity generation and revenue assumed in Eq.(1); therefore, by changing the scale parameter between its minimum and maximum limits, a $24 \%$ change in the revenue is expected, similar to that observed for the average power production in Section 5.2. Assuming a wholesale electricity price of $£ 46.44$ per Megawatt-hour, which is the average of the UK electricity price since 2010 (44) and applying Eq.(1), changing the scale parameter between its maximum 
and minimum limit translates into yearly revenues varying between a minimum of $£ 751,237.95$ and a maximum of $£ 949,911.22$ with the original scale and shape parameter resulting in revenue of $£ 859,236.82$.

To calculate financial (material) losses resulting from failure in the FLS, the framework described in Section 3 is applied. Firstly, the probability of failure for the monopile is calculated using the limit state equation, Eq.(5), solved with importance sampling. To this aim, the design point is located in standard normal space using the first order reliability methods (35) and the probability of failure is evaluated by drawing 10,000 random samples about this point. The results are shown in Figure 10 (left) in terms of lifetime probability of failure and indicate that the small values of fatigue damage predicted for the OWT in Section 5.2 result in small probabilities of failure. However, it is notable that the changing damage values (due to the various climate-change scenarios) cause magnified changes in the probability of failure. When moving from the minimum to maximum scale parameter, the probability of failure increases by a factor of 12 , whereas the fatigue damage only increases by a factor of 1.4. However, it should be noted that the considered NREL 5MW OWT has been designed for the purposes of academic studies; therefore, the absolute values of the obtained probability of failures may not be representative of a real OWT.

The loss calculation is finally applied following the procedure described in Section 3.3, which encompasses both the monopile, tower and other non-structural components. The monopile cost is calculated using Eq.(8) using the parameters for the NREL 5MW OWT, with: $\mathrm{P}_{\mathrm{WT}}=5 \mathrm{MW}, h_{\text {water }}=20 \mathrm{~m}, h_{\text {hub }}=87.6 \mathrm{~m}, \phi_{R}=64 \mathrm{~m}$; the resulting cost is $€ 2.38 \cdot 10^{6}$. This is converted into pounds sterling using the average exchange rate in the year 2013 which was 1.1769 , resulting in a cost of $£ 2.80 \cdot 10^{6}$. The resulting expected annual losses for the system, calculated using Eq.(7), are shown in Figure 10 (right). The lifetime probability of failure, from Figure 10 (left) has been converted into an 'equivalent' annual probability of failure (although it should be noted that the actual probability of failure increases yearly, as discussed in (11)) by dividing by the assumed design life of 20years and used to calculate annual loss. In this case, varying the scale parameter between its minimum and maximum values increases the annual losses by less than $0.1 \%$. This is because the absolute value of lifetime fatigue damage is low and, therefore, the resulting probability of FLS failure is low as well. This means that the structural components have a low weight in the loss calculation, as the loss is dominated by the other non-structural components with higher failure rates such as the gearbox (see Table 2), as indicated by the plane in Figure 10 (right) which shows the total loss when the structural FLS is neglected. This can be seen more clearly in Figure 11 where the complementary cumulative distribution function for the annual losses is plotted, it shows the difference between equipment only and including structural components in the loss calculation. However, it is important to note that failure of a main structural component would result in loss of the generation ability and would ultimately cause loss in revenue for the entire OWT.

A comparison between all the important variables used to calculate the production revenue and loss are shown on Figure 12 in terms of a ratio between the parameter calculated for different scale and shape parameters to the site original scale and shape parameter (described in Section 4.2). The figure shows the constant scaling between power and revenue. It also shows how 
fatigue damage is magnified into probability of failure and again into revenue losses (due to loss of the turbine) but reduced when only system material losses are considered.
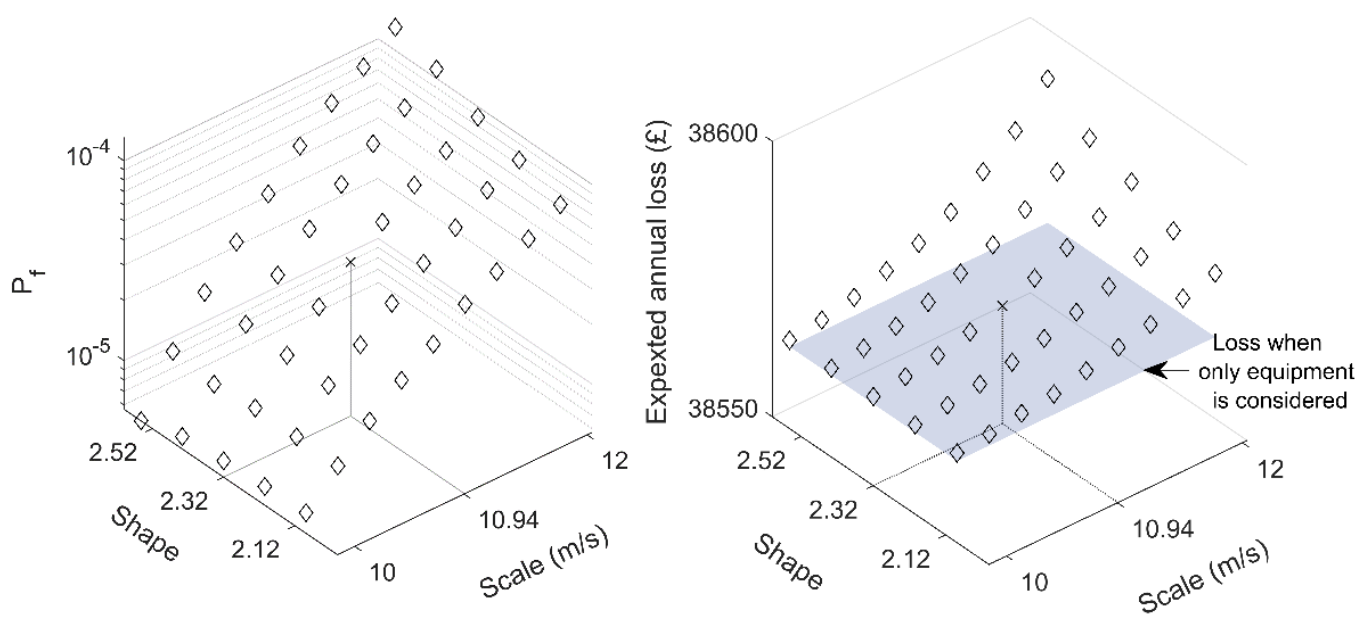

Figure 10: Probability of failure (left) and annual financial losses (right) for various values of the scale and shape parameters of the mean wind speed distribution. The plane shows the material losses when the structural components are neglected.

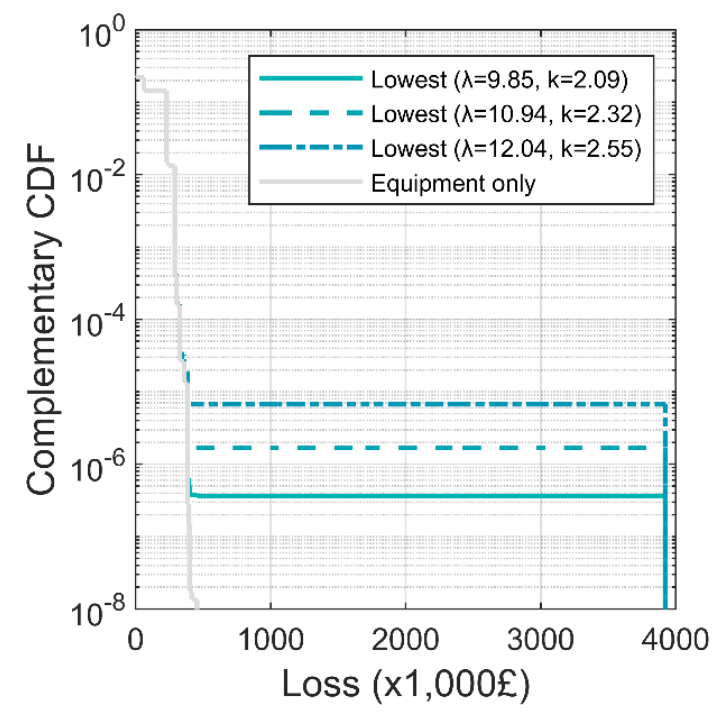

Figure 11: Loss complementary cumulative distribution function for the OWT, showing the difference between the equipment only and including the structural component. 


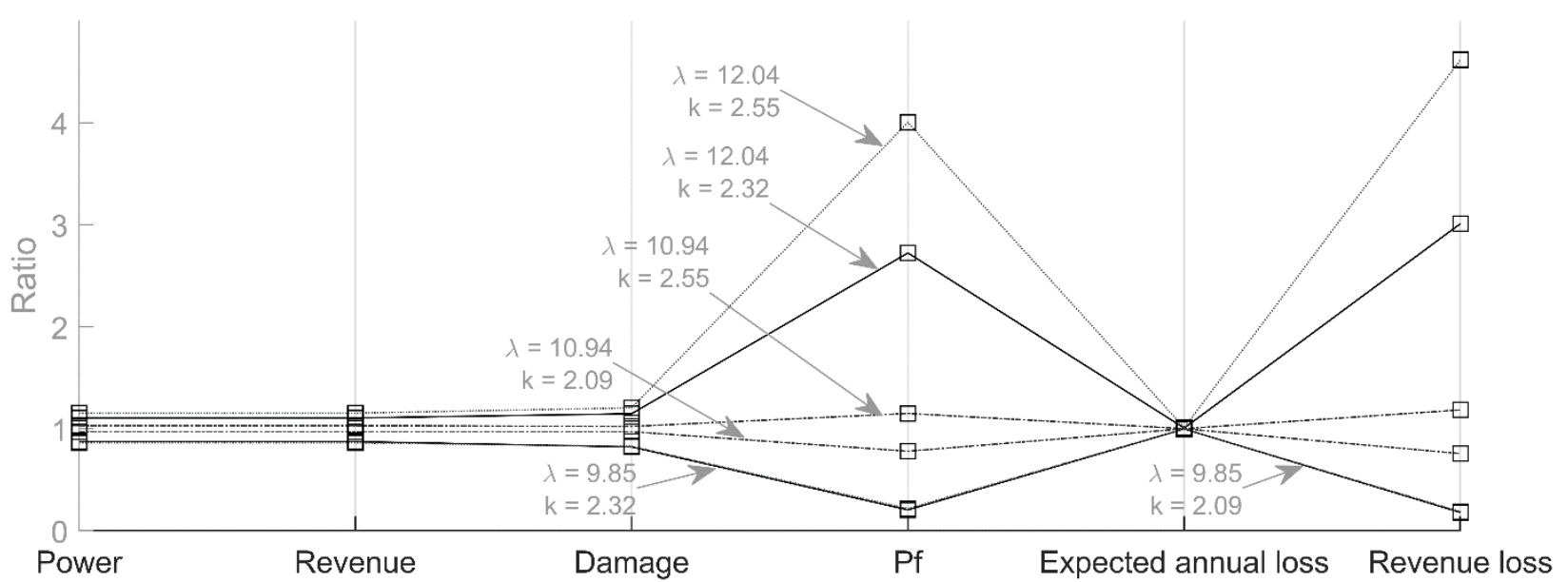

Figure 12: Ratio of the different performance metrics considered in this study calculated at the indicated value of scale and shape parameter to the site scale and shape parameter.

\section{Conclusions}

This study has presented a simulation-based, engineering approach to assess various performance metrics associated with offshore wind turbines exposed to operational wind and wave conditions. A surrogate-modelling approach has been employed to predict structural failure due to the fatigue limit state in a computationally efficient manner. The proposed surrogate model has been implemented in a probabilistic performance-based assessment framework for OWTs that considers both structural and non-structural (equipment) components. Such a framework has been used to evaluate the potential impact of climatechange scenarios on various performance metrics, namely, fatigue damage, fatigue reliability, financial losses (cost of direct damage), and revenue resulting from power production for a case study OWT. Specifically, climate-change impact on OWT loading has been assessed directly in terms of wind loading and through the correlation between this variable and the other important environmental conditions, such as wave height.

Both fatigue damage and structural safety were found to be sensitive to changes in the site environmental conditions. However, as financial material losses additionally depend on non-structural components - which are typically characterised by much higher failure rates - they are found to be less sensitive to the considered climate-change scenarios. In particular, it was found that the monopile has an almost negligible influence on the computed financial losses even although the cost of structural failure would be much larger than that of the other components. This is a result of the low fatigue failure rate predicted for the case-study NREL 5 MW OWT. However, the sensitivity of fatigue damage and resulting probability of failures to changes in wind climate is still relevant to OWT design, and particularly if OWT life extension is of concern. In fact, the predicted fatigue damage due to various climate-change scenarios may be higher than that resulting from optimised design procedures. Additionally, the revenue losses due to failure of the OWT dominates material losses. 
It should be noted that these findings were based on parametric assumptions about the possible impact of climate change on the wind speed distribution at a case-study site, and not on an explicitly modelling of climate change and its effects on the occurring wind speed values. This approach was judged to be suitable given that a range of different climate change scenarios are predicted by various studies depending on the location of the offshore wind farm and that the relative change in power production and fatigue damage were of primary interest.

\section{Acknowledgement}

This work was supported by the UK Engineering and Physical Sciences Research Council (EPSRC), Doctorial Prize Fellowship grant EP/R513143/1 for University College London. The authors acknowledge the use of the UCL Myriad High Throughput Computing Facility (Myriad@UCL), and associated support services, in the completion of this work.

\section{References}

1. Birol F. Offshore wind outlook 2019. 2019.

2. 4C Offshore - Global Offshore Wind Farm Database [Internet]. 2020 [cited 2020 Feb 28]. Available from: https://www.4coffshore.com/windfarms/

3. Vorpahl F, Schwarze H, Fischer T, Seidel M, Jonkman J. Offshore wind turbine environment, loads, simulation, and design. Wiley Interdiscip Rev Energy Environ. 2013;2(5):548-70.

4. Wilkie D, Galasso C. A probabilistic framework for offshore wind turbine loss assessment. Renew Energy [Internet]. 2020 Mar;147:1772-83. Available from: https://linkinghub.elsevier.com/retrieve/pii/S0960148119313734

5. Hubler C, Gebhardt CG, Rolfes R. Methodologies for fatigue assessment of offshore wind turbines considering scattering environmental conditions and the uncertainty due to finite sampling. Wind Energy [Internet]. 2018;(May):114. Available from: http://doi.wiley.com/10.1002/we.2216

6. Jaap S. Fatigue of structures and materials. 2nd ed. Amsterdam: Springer Science + Buisiness Media; 2009.

7. Wilkie D, Galasso C. Site-specific ultimate limit state fragility of offshore wind turbines on monopile substructures. Eng Struct. 2020;204.

8. Morató Casademunt A, Sriramula S, Krishnan N. Reliability analysis of offshore wind turbine support structures using Kriging models. In: Risk, Reliability and Safety: Innovating Theory and Practice: Proceedings of ESREL 2016. Aberdeen; 2016.

9. Teixeira R, Nogal M, O’Connor A, Nichols J, Dumas A. Stress-cycle fatigue design with Kriging applied to offshore wind turbines. Int J Fatigue [Internet]. 2019;125(March):454-67. Available from: https://doi.org/10.1016/j.jfatigue.2019.04.012 
10. Stieng LES, Muskulus M. Reliability-based design optimization of offshore wind turbine support structures using analytical sensitivities and factorized uncertainty modeling. Wind Energy Sci. 2020;5(1):171-98.

11. Wilkie D, Galasso C. Using Gaussian processes in fatigue reliability assessment of offshore wind turbines. Struct Saf.

12. Slot RMM, Sørensen JD, Sudret B, Svenningsen L, Thøgersen ML. Surrogate model uncertainty in wind turbine reliability assessment. Renew Energy. 2019;

13. Murcia JP, Réthoré PE, Dimitrov N, Natarajan A, Sørensen JD, Graf P, et al. Uncertainty propagation through an aeroelastic wind turbine model using polynomial surrogates. Renew Energy. 2018;119:910-22.

14. Solaun K, Cerdá E. Climate change impacts on renewable energy generation. A review of quantitative projections. Renew Sustain Energy Rev. 2019;116.

15. Barstad I, Sorteberg A, Mesquita M dos-SS. Present and future offshore wind power potential in northern Europe based on downscaled global climate runs with adjusted SST and sea ice cover. Renew Energy [Internet]. 2012 Aug;44:398-405. Available from: http://dx.doi.org/10.1016/j.renene.2012.02.008

16. Carvalho D, Rocha A, Gómez-Gesteira M, Silva Santos C. Potential impacts of climate change on European wind energy resource under the CMIP5 future climate projections. Renew Energy. 2017;101(2017):29-40.

17. Tobin I, Jerez S, Vautard R, Thais F, Van Meijgaard E, Prein A, et al. Climate change impacts on the power generation potential of a European mid-century wind farms scenario. Environ Res Lett. 2016;11(3).

18. Soares PMM, Lima DCA, Cardoso RM, Nascimento ML, Semedo A. Western Iberian offshore wind resources: More or less in a global warming climate? Appl Energy [Internet]. 2017;203(2017):72-90. Available from: http://dx.doi.org/10.1016/j.apenergy.2017.06.004

19. Gaetani M, Vignati E, Huld T, Dosio A. Climate modelling and renewable energy resource assessment. 2015;

20. Devis A, Van Lipzig NPM, Demuzere M. Should future wind speed changes be taken into account in wind farm development? Environ Res Lett. 2018;13(6).

21. Hemer MA, Fan Y, Mori N, Semedo A, Wang XL. Projected changes in wave climate from a multi-model ensemble. Nat Clim Chang. 2013;3(5):471-6.

22. Jonkman J, Butterfield S, Musial W, Scott G. Definition of a 5-MW reference wind turbine for offshore system development $\quad$ [Internet]. 2009. Available http://tethysdevelopment.pnnl.gov/sites/default/files/publications/Jonkman_et_al_2009.pdf

23. Burton T, Jenkins N, Sharpe D, Bossanyi E. Wind Energy Handbook Second Edition [Internet]. 2011. 775 p. Available from: http://doi.wiley.com/10.1002/9781119992714

24. Kulkarni S, Deo MC, Ghosh S. Changes in the design and operational wind due to climate change at the Indian offshore sites. Mar Struct. 2014;37:33-53.

25. Rubert T, McMillan D, Niewczas P. A decision support tool to assist with lifetime extension of wind turbines. Renew Energy [Internet]. 2018;120:423-33. Available from: https://doi.org/10.1016/j.renene.2017.12.064

26. Damiani RRR. Design of offshore wind turbine towers [Internet]. Offshore Wind Farms: Technologies, Design and 
Operation. Elsevier Ltd; 2016. 263-357 p. Available from: http://dx.doi.org/10.1016/B978-0-08-100779-2.00010-6

27. Jonkman B, Jonkman J. FAST Readme v8.12.00a-bjj. Denver; 2015.

28. DNV GL. Fatigue design of offshore steel structures. 2016. Report No.: DNV-RP-C203.

29. Sorensen JD, Frandsen S, Tarp-Johansen NJ. Effective turbulence models and fatigue reliability in wind farms. Probabilistic Eng Mech. 2008;23(4):531-8.

30. Carroll J, McDonald A, McMillan D. Failure rate, repair time and unscheduled O\&M cost analysis of offshore wind turbines. Wind Energy [Internet]. 2016 Jun;19(6):1107-19. Available from: http://doi.wiley.com/10.1002/we.1887

31. Kelley ND, Jonkman B. NREL/TP-500-41137. Overview of the turbsim stochastic inflow turbulence simulator. 2007.

32. Jonkman J, Robertson A, Hayman G. HydroDyn user's guide and theory manual. Denver; 2015.

33. DNV GL. Environmental conditions and environmental loads. 2017.

34. Rychlik I. A new definition of the rainflow cycle counting method. Int J Fatigue. 1987;9(2):119-21.

35. Au SK, Wang Y. Engineering risk assessment with subset simulation. Singapore: John Wiley \& Sons; 2014. 315 p.

36. Santner TJ, Williams BJ, Notz WI. The Design and Analysis of Computer Experiments [Internet]. New York, NY: Springer New York; 2003. (Springer Series in Statistics). Available from: http://link.springer.com/10.1007/978-14757-3799-8

37. Huchet Q, Mattrand C, Beaurepaire P, Relun N, Gayton N. AK-DA: An efficient method for the fatigue assessment of wind turbine structures. Wind Energy. 2019;22(5):638-52.

38. Rasmussen C, Williams C. Gaussian processes for machine learning [Internet]. Cambridge: MIT Press; 2006. Available from: http://ieeexplore.ieee.org/xpl/articleDetails.jsp?arnumber=6279024

39. Dicorato M, Forte G, Pisani M, Trovato M. Guidelines for assessment of investment cost for offshore wind generation. Renew Energy [Internet]. 2011;36(8):2043-51. Available from: http://dx.doi.org/10.1016/j.renene.2011.01.003

40. Damgaard M, Andersen LV, Ibsen LB, Toft HS, Sørensen JD. A probabilistic analysis of the dynamic response of monopile foundations: Soil variability and its consequences. Probabilistic Eng Mech [Internet]. 2015;41:46-59. Available from: http://www.sciencedirect.com/science/article/pii/S0266892015300151

41. FINO project homepage [Internet]. 2020 [cited 2020 Mar 9]. Available from: https://www.fino-offshore.de/en/

42. Hübler C, Gebhardt CG, Rolfes R. Development of a comprehensive data basis of scattering environmental conditions and simulation constraints for offshore wind turbines. Wind Energy Sci. 2017;2(2015):491-505.

43. Zwick D, Muskulus M. The simulation error caused by input loading variability in offshore wind turbine structural analysis. Wind Energy [Internet]. 2015;18(8):1421-32. Available from: http://dx.doi.org/10.1002/we.1767

44. Electricity prices: Day-ahead baseload contracts - monthly average (GB) [Internet]. 2019 [cited 2020 Mar 3]. Available from: https://www.ofgem.gov.uk/data-portal/electricity-prices-day-ahead-baseload-contracts-monthlyaverage-gb 